\title{
Stochastic Analysis of the Effect of Asset Prices to a Single Economic Investor
}

\author{
E. Ekuma-Okereke $^{1 *}$, J. T. Eghwerido ${ }^{1}$, E. Efe-Eyefia ${ }^{1}$, S. Zelibe ${ }^{1}$ \\ ${ }^{1}$ Department of Mathematics/Computer Science \\ Federal University of Petroleum Resouces, P.M.B 1221, Effurun, Delta State, Nigeria
}

*Correspondence: ekuma.okereke@fupre.edu.ng

\author{
Keywords: Risk Neutrality, Dynamic Programming Principle, geometric Brownian motion, \\ Stochastic Control.
}

\begin{abstract}
In this paper, we propose a single economic investor whose asset follows a geometric Brownian motion process. Our objective therefore is to obtain the fair price and the present market value of the asset with an infinitely horizon expected discounted investment output. We apply dynamic programming principle to derive the Hamilton Jacobi Bellman (HJB)-equation associated with the problem which is found to be equivalent to the famous Black-Scholes Model under no risk neutrality. In addition, for a complete market under equilibrium, we obtained the value of the present asset with risk neutrality and its fair price.
\end{abstract}

\section{Introduction}

The study that Asset Prices follow a simple diffusion process was first proposed in the historical work of Bachelier in 1900. His work is rather remarkable in that it addressed the problem of option pricing and his main aim was to actually provide a fair price for the European call option [3]. Bachelier assumed stock price dynamics with a Brownian motion without drift (resulting in a normal distribution for the stock prices), and no time-value of money. The formula provided may be used to valuate a European style call option [10]. Later on, [4] obtained the same results as Bachelier. As pointed out by [5] and [9], this approach allows negative realizations for both stock and option prices. Moreover, the option price may exceed the price of its underlying asset.

According to [10], Black - Scholes Model is often regarded as either the end or the beginning of the option valuation history. Using two different approaches for the valuation of European style options, they present a general equilibrium solution that is a function of "observable" variables only, making therefore the model subject to direct empirical tests. The stock price dynamics is described by a geometric Brownian motion with drift as a more refined market model for which prices cannot be negative, the volatility can be viewed as the diffusion coefficient of this random walk [7]. The manifest characteristic of the final valuation formula is the parameters it does not depend on. The option price does not depend on the expected return rate of the stock or the risk preferences of the investors. It is not assumed that the investors agree on the expected return rate of the stock. It is expected that investors may have quite different estimates for current and future returns. However, the option price depends on the risk-free interest rate and on the variance of the return rate of the stock.

To make simplier normally distributed financial asset returns, on assumption that its distribution is difficult, [11] concludes that for a distribution model to reproduce the properties of empirical evidence it must possess certain parameters as: a location parameter; a scale parameter; an asymmetry parameter and a parameter describing the decay of the distribution since financial returns distribution is difficult to determine since normally distributed financial asset returns is not supported by empirical evidence.

We suppose the movement of an asset price is a stochastic process, thus the price paths of stocks and indices will all be modeled using this idea. The stochastic process followed by the underlying asset is considered in a continuous time. Our concern therefore is to construct the stochastic control problem to emerge from solving the infinitely horizon expected discounted investment output. While existence and closed form solution to the stochastic control problem 
follows from well-known theory, it is also found to be equivalent to the famous Black-Scholes Model under no risk neutrality, though the market is complete. Interestingly, our proposed method have been successfully used by many authors, see for examples $[8,13]$.

\section{Problem formulation}

We reformulate the works of [6] and [14] for a stock market with the following properties. Uncertainty is represented by a complete filtered probability space $\left(\Omega, f,\left(f_{t}\right), P\right)$ and throughout the paper, we denote by $\left(f_{t}\right)_{t \geq 0}$ the neutral filtration i.e. $f_{t}=\sigma\{W(s) ; 0 \leq s \leq t\}$ where $(W()$.$) is a$ standard 1-dimensional Brownian motion defined on this space with value in $R$.Consider an investor whose underlying risky asset (e.g. stock) follows the setup below. Let $S(t)$ be the unit price for the risky asset assume to follow a geometric Brownian motion process. Given the above assumptions, the dynamics of the risky asset is:

$$
\frac{d S(t)}{S(t)}=\mu d t+\sigma d W(t), S(0)>0
$$

such that the solution of equation (1.1) in ito's sense is given by:

$$
\frac{S(t)}{S(0)}=\exp \left[\left(\mu-\frac{1}{2} \sigma^{2}\right) t+\sigma W(t)\right], \quad \forall t \geq 0
$$

where $\mu, \sigma$ are the drift and volatility parameters assume to be positive constants and $W(t)$ is a standard 1-dimensional Brownian motion [12].

Fair price of an asset for an infinite investment is considered in this paper. The Objective function as formulated in [6] of the investor is the infinite horizon expected discounted investment output given by:

$$
V(S(t), k(t), t)=\operatorname{Sup}_{u>0} \int_{t}^{\infty} e^{-r t}[P(S(t), k(t))-u(S(t), k(t), t) C(S(t), k(t))] d t, t \geq 0
$$

subject to the stochastic control variable $u(S(t), k(t), t)$ given by

$$
u d t=d k(t) \quad k(0)>0, u \geq 0
$$

where $k(t) \in[0, \infty)$ is the capital and the stochastic control parameter to be controlled by the economic investor, $P(S(t), k(t))$ is the production rate and $C(S(t), k(t))$ is the cost of expansion in the form of liabilities and $r$ is the discount rate term.

Using the dynamic programming principle, the Hamilton-Jacobi-Bellman (HJB) -equation corresponding to (1.2) is given by:

$$
\underset{u \geq 0}{\operatorname{Sup}}\left\{\frac{\partial V}{\partial t}+\mu S \frac{\partial V}{\partial S}+\frac{1}{2} \sigma^{2} S^{2} \frac{\partial^{2} V}{\partial S^{2}}+u \frac{\partial V}{\partial k}+(P-u C) e^{-r t}\right\}=0 .
$$

If

$$
\frac{\partial V}{\partial k}=C e^{-r t}
$$

then, (1.4) becomes

$$
\frac{1}{2} \sigma^{2} S^{2} \frac{\partial^{2} V}{\partial S^{2}}+\mu S \frac{\partial V}{\partial S}-r V=-P
$$


Lemma. Consider a portfolio

$$
\{H(t)\} t \geq 0
$$

which is $F_{t}$-measurable and $H(t)$ denotes the number of shares that the investor is holding at time $t$. If the stock price $S(t)$ follows a geometric BM

$$
d S(t)=\mu S(t) d t+\sigma d W(t)
$$

with wealth of the investor as

$$
d X(t)=[r X+H S(\mu-r)] d t+H S \sigma d W(t) .
$$

Suppose that the value of the claim at time $t$ is given by

$$
G(t)=V(S, t)
$$

then

$$
\left[\frac{\partial V}{\partial t}+\mu S \frac{\partial V}{\partial S}+\frac{1}{2} \sigma^{2} S^{2} \frac{\partial^{2} V}{\partial S^{2}}\right] d t+\sigma S \frac{\partial V}{\partial S} d W=d G(t)
$$

such that

$$
r S \frac{\partial V}{\partial S}+\frac{1}{2} \sigma^{2} S^{2} \frac{\partial^{2} V}{\partial S^{2}}-r V=-\frac{\partial V}{\partial t}
$$

where(1.12) is the famous B-S Model.

Proof. The resulting relation by equating (1.9) and (1.11) gives:

$$
\frac{\partial V}{\partial t}+\mu S \frac{\partial V}{\partial S}+\frac{1}{2} \sigma^{2} S^{2} \frac{\partial^{2} V}{\partial S^{2}}=r X+H S(\mu-r)
$$

and

$$
\sigma S \frac{\partial V}{\partial S}=H S \sigma
$$

with

$$
H=\frac{\partial V}{\partial S}(S, t)
$$

If $X(t)=G(t)=V(S, t)$, then with equations (1.14) and (1.15), equation(1.13) becomes:

$$
r S \frac{\partial V}{\partial S}+\frac{1}{2} \sigma^{2} S^{2} \frac{\partial^{2} V}{\partial S^{2}}-r V=-\frac{\partial V}{\partial t} .
$$

\section{Solution of the asset model and its unique price with no risk neutrality}

The time-homogenous form of (1.6) is equivalent to (1.16) such that

$$
\frac{1}{2} \sigma^{2} S^{2} \frac{\partial^{2} V}{\partial S^{2}}+\mu S \frac{\partial V}{\partial S}-r V=-\frac{\partial V}{\partial t}
$$

this implies

$$
\frac{\partial V}{\partial t}(S(t), K(t), t)=P(S(t), K(t))
$$


Applying [2], the rate of change of investment output is with respect to $S$, then we have (2.2) rewritten as:

$$
\frac{d V}{d t}=P(S)=S
$$

By (2.3), equation (2.1) therefore reduces to ordinary differential equation of the form:

$$
\frac{1}{2} \sigma^{2} S^{2} \frac{\partial^{2} V}{\partial S^{2}}+\mu S \frac{\partial V}{\partial S}-r V=-S
$$

\section{Main Result}

Theorem. Let $\left(\Omega, f,\left(f_{t}\right), P\right)$ be a complete filtered probability space and let $V(S)$ be the investment output of a single economic investor and let $S$ be the stock price. Suppose that $V(S)$ is twice continuously differentiable generated by $V(0)=0, \frac{d V}{d S}=0$, then the solution of (2.4) found to be

$$
V(S)=A S^{m_{1}}+\frac{S}{(r-\mu)}
$$

and the unique equilibrium stock price is

$$
\hat{S}=\frac{m_{1}(r-\mu) \bar{S}}{m_{1}-1}
$$

where $m_{1}=-\left(\mu-\frac{\sigma^{2}}{2}\right)-\left\{\left(\mu-\frac{\sigma^{2}}{2}\right)^{2}+2 \sigma^{2} r\right\}^{\frac{1}{2}}$ is the negative characteristics root of $\operatorname{cor}(\sigma, r)=m_{1}<0$, and $\mathrm{A}$ is a constant.

Proof of Theorem. Applying the method used by [8] and setting $S=e^{t},(2.4)$ becomes

$$
\frac{\sigma^{2}}{2} D^{2} V-\frac{\sigma^{2}}{2} D V+\mu D V-r V=-e^{t}
$$

Where $D=\frac{d}{d S}$.

It is easy to see that the positive and negative roots of the characteristics solution from equation (2.8) is

$$
m_{1} m_{2}=-\left(\mu-\frac{\sigma^{2}}{2}\right) \pm\left\{\left(\mu-\frac{\sigma^{2}}{2}\right)^{2}+2 \sigma^{2} r\right\}^{\frac{1}{2}}
$$

With the correlation coefficient given as $\mu$ and the characteristics and particular solutions are therefore given as:

$$
\begin{array}{r}
V_{c}=A e^{m_{1} t}+B e^{-m_{2} t} \\
V_{p}=C e^{t}=\frac{e^{t}}{(r-\mu)}
\end{array}
$$


Where $C=\frac{1}{(r-\mu)}$. (A, B, C are constants).

The general solution now becomes:

$$
V(S)=A S^{m_{1}}+\frac{S}{(r-\mu)} .
$$

Under equilibrium condition, the fair price $\hat{S}$ must be equal to the expected cost $\bar{S}$ of the risky asset [8]. We therefore have

$$
V(\hat{S})=A m_{1} \hat{S}^{m_{1}}+\frac{\hat{S}}{(r-\mu)}=\bar{S}
$$

solving for $\hat{S}$, we have

$$
\hat{S}=\frac{m_{1}(r-\mu) \bar{S}}{m_{1}-1}
$$

Remark: We considered the problem on the assumption that the market is an incomplete market. Therefore the drift parameter regarded as $\mu$ is not equal to the discounted rate parameter regarded as $r$ so that the fair price $\hat{S}$ does not tend to zero.

\section{Unique asset price under risk neutrality}

In equation (1.12), the drift term $\mu$ disappears. This follows from the idea of risk-neutral pricing by hedging with no possibility of arbitrage [1]. It will be shown that the drift on an underlying asset must be considered risk-neutral regardless of the pricing method used. Therefore, to fairly price asset, consider

$$
r S \frac{\partial V}{\partial S}+\frac{1}{2} \sigma^{2} S^{2} \frac{\partial^{2} V}{\partial S^{2}}-r V=-\frac{\partial V}{\partial t}
$$

which follows risk-neutral random work.

To derive the fair asset price from equation (3.1), we make the following change of variables:

$$
U=e^{-r t} V
$$

and

$$
W=e^{-r t} S
$$

whichleads to:

$$
\frac{\partial V}{\partial S}=\frac{U}{W}
$$

By substitution of equation (3.4) into equation (3.1), it becomes:

$$
\frac{1}{2} \sigma^{2} W^{2} \frac{\partial^{2} U}{\partial W^{2}}=-\frac{\partial U}{\partial t}
$$

By inspection, equation (3.5) has been reduced to a parabolic type of equation with boundary conditions having eliminated the interest rate term. Hence, by separation of variables method let the solution be given as:

$$
U=W(w) T(t)
$$


with

$$
U(w, t)=0
$$

and

$$
\frac{\partial U}{\partial W}(w, t)=0, \forall t
$$

Differentiating and substituting equation (3.6) into equation (3.5) gives:

$$
\frac{1}{W} \frac{\partial^{2} W}{\partial w^{2}}=-\frac{1}{K^{2} T} \frac{\partial T}{\partial t}
$$

since the LHS and RHS of equation (3.9) are functions of w and t respectively, then they can be equated to the same constant. We then write:

$$
\frac{1}{W} \frac{\partial^{2} W}{\partial w^{2}}=-\frac{1}{K^{2} T} \frac{\partial T}{\partial t}=\lambda
$$

where $\lambda$ is arbitrary constant and $K^{2}=2(\sigma w)^{-2}$.

Therefore, the solutions of equation (3.10) becomes

$$
W(w)=e^{m w}
$$

and

$$
T(t)=C e^{-\lambda k^{2} t}
$$

where $m= \pm \sqrt{\lambda}$ is the characteristics roots of the LHS of equation (3.10) and $C$ is a constant.

Putting (3.11) and (3.12) into (3.6), the solution becomes:

$$
U(w, t)=C e^{m w-\lambda k^{2} t}
$$

where $k^{2}=2(\sigma w)^{-2}$.

Thus

$$
U(w, t)=e^{m w-2 \lambda(\sigma w)^{-2} t}
$$

The constant $C$ can be absorbed, so that we now apply the boundary conditions.

$$
\frac{\partial U}{\partial w}=\left(m+4 \lambda \sigma^{-2} w^{-3} t\right) e^{m w-2 \lambda(\sigma w)^{-2} t}=0
$$

solving for $w$, have

thus,

$$
m+4 \lambda \sigma^{-2} w^{-3} t=0
$$

$$
W=-\sqrt[3]{\frac{4 \lambda \sigma^{-2} t}{m}} .
$$

Equating (3.16) with (3.3) we have:

$$
S=\left(-\sqrt[3]{\frac{4 \lambda \sigma^{-2} t}{m}}\right) e^{r t},
$$

Similarly, equation (3.14) with equation (3.2) gives:

$$
V=e^{\sqrt{\lambda} s-2 \lambda(\sigma)^{-2} t+r t}
$$




\section{Conclusions}

In this paper, we considered a stochastic control problem of a singled economic investor. The fair prices and its values (output) under the assumptions of risk neutrality and non-risk neutrality were both obtained in an infinitely horizon expected discounted investment output. If $\mu>0, \sigma>0, r>0$ with $(r-\mu)>0$ then $m_{1}<0$ such that $\hat{S}$ of (2.14) is finite. Also if $\mu-\frac{\sigma^{2}}{2} \rightarrow 0$ then $m_{1}<0$ such that $\hat{S}$ of (2.14) is finite, $\mu-\frac{\sigma^{2}}{2} \rightarrow \infty$ then $m_{1}<0$ such that $\hat{S}$ of (2.14) is finite. The equilibrium asset price $\hat{S}$ remains negative for $m_{1} \in(0,1)$. If $m_{1}=1$ we obtain indeterminate solution. For positive fair price with $(r-\mu)>0$ returns a positive and increasing value in asset while for $(r-\mu)<0$ returns a negative and decreasing value in asset. In addition growth in ouput (3.18) is affected by the condition that $m_{1}<0$.

\section{References}

[1] A.D. Andricopoulos, Option Pricing Using Quadrature and other Numerical Methods, Ph.D. thesis, Department of Mathematics, Manchester United, 2002.

[2] J. Busca, Introduction to Financial Mathematics UNICAMP, Campinas, Brazil, 2002.

[3] J.C. Hull, Options, Futures, and other derivatives, Prentice Hall, New York, 1997.

[4] R.J. Kruizenga, Put and call options: A theoretical and market analysis, Ph.D. Thesis, Cambridge, MA: MIT, 1956.

[5] R.C. Merton, The theory of rational option pricing, The Bell Journal of Economics and Management Science. 4 (1973) 141-183.

[6] S.E. Onah, O.O. Ugbebor, Solution of a Two-dimensional Stochastic Investment Problem, J. App. Math. Comp. 98 (1999) 75-82.

[7] M. F. M. Osborne, Brownian motion in the stock market, Operations Research. 7(2) (1959) $145-173$.

[8] B.O. Osu, G.U. Achi, C.A. Emereuwa, Stochastic (Multiplicative) Effect of Government Policy on the income of individuals, J. Modern Mathematics and Statistics. 5(1) (2011) 3-8.

[9] C. W. Smith, Option pricing: a review, Journal of Financial Economics. 3 (1976) 3-52.

[10] S.R. Straja, Stochastic Modeling of Stock Prices, Montgomery Investment Technology, Inc, Camden, NJ 08103, 1997.

[11] R. Cont, Empirical Properties of Asset Returns. Stylized facts and Statistical Issues, Quantitative Finance. 1 (2001) 223 -236.

[12] W. Huang, et al., The optimal Fuzzy Portfolio Strategy with Hedging, Theoretical Mathematics and Application. 2(1) (2012) 1-12.

[13] A.C. Okoroafor, B.O. Osu, A Stochastic iteration Method for the Solution of finite dimensional variation inequalities, J. Nig. Association of Mathematical Physics. 8 (2004) 301-304. MR 1952680

[14] E. Ekuma-Okereke, B.O. Osu, Dynamic Optimization of Portfolios with Tail Conditional Expectations (TCE) Constraints, Asian Journal of Mathematics and Applications. 2013 (2013). 